\title{
Computational corneal biomechanics in the clinic
}

Miguel Ángel Ariza-Gracia',2, David Pablo Piñero Llorens², José Félix Rodríguez Matas $^{4}$, Begoña Calvo Calzada ${ }^{1,5}$

${ }^{1}$ Applied Mechanics and Bioengineering (AMB), Aragón Institute for Engineering Research (i3A), University of Zaragoza, Zaragoza, Spain; 'Institute for Surgical Technology and Biomechanics, University of Bern, Bern, Switzerland; ${ }^{3}$ OFTALMAR, Vithas Medimar International Hospital, Alicante, Spain; ${ }^{4}$ LaBS, Politecnico di Milano (Polytechnic University of Milan), Milan, Italy; ${ }^{5}$ Center for Biomedical Research in Bioengineering, Biomaterials, and Nanomedicine (CIBER-BBN), Madrid, Spain

\section{Abstract}

Corneal topographers and air-puff devices aim at completely characterizing so-called corneal biomechanics, a collection of features that describes corneal behavior. The European FP7 project (PopCorn) was born with the goal of integrating both technologies. Among the novelties, computational models were included as an integral part of the clinical assessment. Automatic patient-specific (P-S) reconstruction of the cornea, alongside material prediction based on finite element simulations, optimization, and fitting were used to strive forward in a priori surgical planning. Both methodologies show good performance in retrieving the P-S geometry of the cornea (error $<1 \%$ ) and the maximum deformation amplitude of a non-contact tonometry (error 5\%). Nevertheless, physiological and non-physiological corneas cannot be classified solely in terms of material, at least with a single experiment. Eventually, and due to the interplay of different factors (geometry, material, and pressure), results coming from air-puff devices should be handled with care.

Keywords: corneal biomechanics, material characterization, non-contact tonometry, patient-specific

Correspondence: M.Á. Ariza-Gracia, Edificio i3A, Campus Río Ebro Calle Mariano Esquillor Gómez s/n, C.P. 50.018, Zaragoza, Spain.

E-mail:mariza@unizar.es 


\section{Introduction}

Corneal biomechanics is an emerging topic in ophthalmology. Non-contact tonometers aim at determining the intraocular pressure of the eye (IOP) and characterizing the mechanical properties of corneal tissue by applying a short air jet (approximately $20 \mathrm{~ms}$ ) on the cornea. Different corneal biomarkers are recorded as the cornea deforms, establishing an interesting framework to determine the mechanical properties of the corneal tissue by means of inverse optimization processes. ${ }^{1}$ The PopCorn European project was born as an effort to combine non-contact tonometers, imaging techniques (i.e., plenoptic imaging), and computational methods to reconstruct the P-S geometry and predict the P-S material. The data from this project will be essential to reach the ultimate objective: personalized surgical simulations.

\section{Material and methods}

First, a theoretical in-silico study on an average cornea was designed to shed light on the interaction between different corneal features (geometry, material, and IOP) (Fig. 1, upper left). ${ }^{2}$ Second, two algorithms were developed: an automatic P-S reconstruction of the cornea using clinical data ${ }^{3}$ and an automatic P-S material predictor based on clinical biomarkers ${ }^{4}$ (Fig. 1, bottom panels). The geometric algorithm reconstructed the 3-D finite element (FE) model including: the patient's topography (i.e., point cloud describing the corneal geometry); an average orthogonal distribution of collagen fibers; the anisotropic hyperelastic response of the corneal tissue (i.e., a non-linear isotropic behavior related to the extracellular matrix, plus an exponential orientation-dependent anisotropic behavior related to the fibers); ${ }^{5}$ and a free-stress algorithm that allows for recovering the natural pre-stress due to the IOP. Furthermore, the automatic non-supervised pipeline allows integrating a geometrical P-S simulation after the reconstruction process (e.g., a non-contact tonometry or surgery). The material predictor combined our former P-S geometric algorithm with in-silico simulations of a non-contact tonometry to sweep a range of P-S geometries, IOPs, and material properties (i.e., in the framework of anisotropic elasticity), building a dataset of $\sim 9.000 \mathrm{FE}$ simulations. The dataset contained the mechanical response of the cornea to inflation experiments in humans ${ }^{6}$ and the kinematic response of the cornea to an air puff. ${ }^{7}$ After filtering those by physiological response (Fig. 1, upper right), the predictors of the material model were set using four mathematical strategies: support vector regression (SVR), multiple layer perceptron (MLP), quadratic response surface (QRS), and a clustering technique based on the $\mathrm{K}$ nearest neighbors (K-nn). Once the models were trained, three clinical biomarkers were used to predict the three material constants of our proposed model: ${ }^{4}$ the maximum corneal displacement of the non-contact 


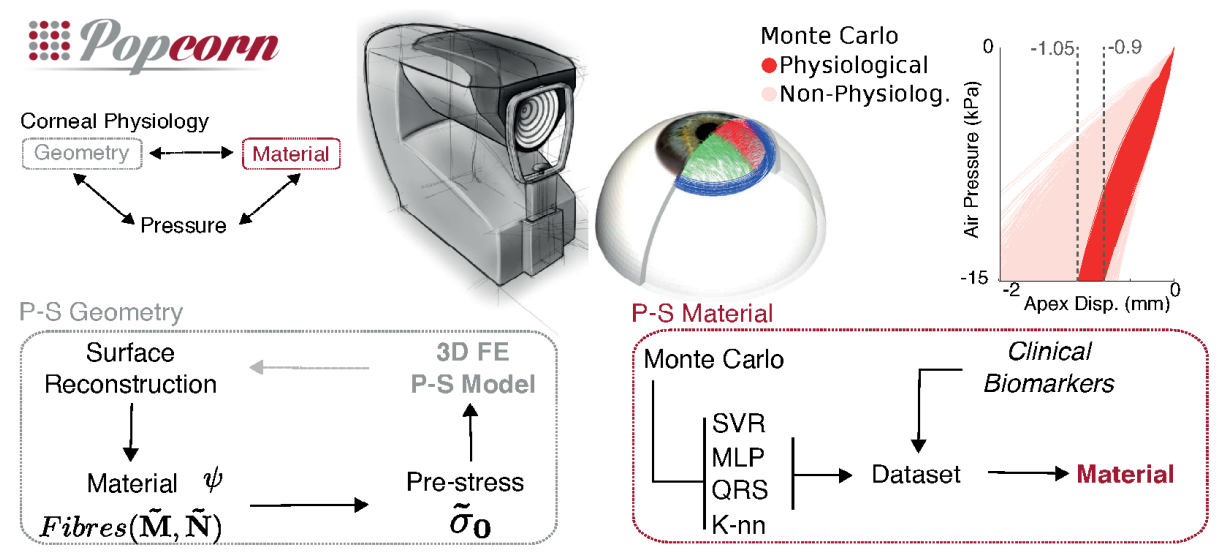

Fig. 1. Methodology pipeline (upper left). Interplay between corneal features (bottom left). Patient-specific geometric reconstruction (bottom right). Patient-specific material prediction (upper right). Monte Carlo simulations for the normal population. Physiological behavior after filtering (dark red) vs non-physiological behavior (light red). Topographer concept (center).

tonometry test (Umax), the corneal thickness (CCT), and the IOP. To validate our protocol, we performed a closed-loop validation using new unknown healthy and diseased real cases. First, the material parameters were predicted based on real clinical biomarkers (Umax, IOP, and CCT). Second, the automatic reconstruction algorithm reconstructed the P-S model (including IOP, geometry, and predicted material properties). Third, we performed the simulation of a generic non-contact tonometer to obtain the simulated displacement (Unum). Finally, the accuracy of the protocol was determined by the difference between both displacements, actual (Umax) and numerical (Unum).

\section{Results}

First, we highlight that an interplay exists between different corneal features (CCT, IOP, and material stiffness) when an air-puff diagnosis test is applied. The maximum apical displacement presents an inverse linear relation with IOP, but an inverse cubic relation with CCT. Meaning that, for similar geometrical features, a compliant (soft) cornea with a high IOP could behave as a stiff cornea with a low IOP. ${ }^{2}$ Second, the automatic geometric algorithm showed that the accuracy of the P-S reconstruction had an error of less than $1 \%$. Disregarding the free-stress configuration of the eye could lead to up to a $10 \%$ of error in the prediction of the displacement (Unum). Not only that, but the sensitivity analysis using P-S features also showed an interplay between the aforementioned variables, supporting the 
outcomes outlined with average models. ${ }^{3}$ Third, regarding the material predictor, a Monte Carlo approach requires significant computational resources to build a reliable dataset, but at the same time, provides great flexibility. After filtering the data to those satisfying the physiological response in inflation and air-puff tests, the distribution of the material parameters was not parametric (i.e., not normal). Moreover, when studying the combinations of material parameters, IOP, and CCT that lead to the same displacement (Unum), the result demonstrates the interplay between parameters: different combinations of IOP and CCT gave the same response depending on the material. Finally, the average validation error of the methodology (i.e., the difference between numerical and real displacement) was approximately $5 \%$, even for diseased eyes (i.e., eyes with keratoconus). ${ }^{4}$

\section{Conclusion}

A computational tool applicable to clinical situations was developed. From P-S reconstruction to the prediction of corneal tissue stiffness, all clinical requests were covered. Due to the interplay of different corneal features (IOP, geometry, and material), physiological and non-physiological eyes cannot be differentiated solely in terms of material, at least not with a single diagnostic test. Therefore, results from non-contact tonometry tests must be handled with care to avoid misdiagnosing. However, this work is not exempt of limitations: we do not include viscoelastic effects, since the test is extremely quick; ${ }^{8} \mathrm{P}$-S distribution of fibers is disregarded, since nowadays it is unlikely to obtain them in the clinic; the internal structures of the eye (i.e., lens and ciliary muscles) are disregarded, the internal humors are treated as a uniform pressure applied over the inner surface of the eyeball, and do not account for mass nor compressibility; and the simulations are assumed to be quasi-static. Next steps should involve a more complex behavior of the corneal tissue, and fluid-structure simulations, currently in the final stages of development. Nevertheless, as the proposed methodology presents a block-wise design, all these features could be introduced without modifying the pipeline.

\section{Acknowledgements}

This work was funded by the Spanish government (DPI201454981R) and the European Union's Seventh Framework (Grant Agreement FP7-SME-2013 606634). Miguel Ángel Ariza-Gracia is supported by the ESKAS program (ESKAS-No. 2016.0194; Federal Commission for Scholarships for Foreign Students FCS, Switzerland). 


\section{References}

1. Piñero DP, Alcón N. Corneal biomechanics: A review. Clin Exp Optom. 2015;98(2):107-116.

2. Ariza-Gracia MÁ, Zurita JF, Piñero DP, Rodriguez-Matas JF, Calvo B. Coupled biomechanical response of the cornea assessed by non-contact tonometry. A simulation study. PLoS One. 2015;10(3):e0121486.

3. Ariza-Gracia MÁ, Zurita J, Piñero DP, Calvo B, Rodríguez-Matas JF. Automatized patient-specific methodology for numerical determination of biomechanical corneal response. Ann Biomed Eng. 2016;44(5):1753-1772.

4. Ariza-Gracia MÁ, Redondo S, Llorens DP, Calvo B, Rodriguez-Matas JF. A predictive tool for determining patient-specific mechanical properties of human corneal tissue. Comput Methods Appl Mech Eng. 2016;317:226-247.

5. Pandolfi A, Holzapfel GA. Three-dimensional modeling and computational analysis of the human cornea considering distributed collagen fibril orientations. J Biomech Eng. 2008;130(6):61006. doi:/10.1115/1.2982251.

6. Elsheikh A, Alhasso D, Rama P. Biomechanical properties of human and porcine corneas. Exp Eye Res. 2008;86(5):783-790.

7. Huseynova T, Waring 4th GO, Roberts C, Krueger RR, Tomita M. Corneal biomechanics as a function of intraocular pressure and pachymetry by dynamic infrared signal and Scheimpflug imaging analysis in normal eyes. Am J Ophthalmol. 2014;157(4):885-893. doi:/10.1016/j.ajo.2013.12.024.

8. Simonini I, Angelillo M, Pandolfi A. Theoretical and numerical analysis of the corneal air puff test. J Mech Phys Solids. 2016;93:118-134. doi:/10.1016/j.jmps.2016.04.012. 